\title{
Research on Drift Path Prediction Technology of Ships
}

\author{
Dongdong $\mathrm{An}^{1}$, Tianchi Zhang ${ }^{1}$, Jing Zhang ${ }^{1,2, *}$, Zhimin $\mathrm{Liu}^{1}$ and Jiasen Chen ${ }^{1}$ \\ ${ }^{1}$ College of computer science and technology, Harbin Engineering University, Harbin, 150001, China \\ ${ }^{2}$ College of Engineering, Shantou University, Guangdong, 515063, China. \\ ${ }^{*}$ Corresponding author
}

\begin{abstract}
In order to improve the prediction fitting degree of the existing method to predict the drift path of the ship, the particle reloading simulation method was proposed to predict the drift path from the aspect of segment prediction. Particle reloading simulation method divides the drift time into multiple segments, and then the information is updated after each segment The updating will continue the whole drift process, and then the drift path will be obtained. Experimental results indicate that our method is extremely promising for predicting drift paths of ships. This is due to the avoidance of the problem of the Monte Carlo method, which prediction accuracy of the long-distance decreases with time.
\end{abstract}

Keywords-drift path; particle reload simulation; prediction degree; Monte Carlo

\section{INTRODUCTION}

With the prosperity of maritime transport, there is the sea accident.The incidence of maritime accidents has been increasing gradually, which has attracted people's attention. Because the accident occurs on the sea, it is very difficult to carry out the rescue. After accidents happen, we cannot rescue real-time at sea, then the wrecked ship will drift over time, and the rescue will not be easy to obtain good results. If we can predict the drift path of the wrecked ship, we can gain more time for the rescue, and minimize the loss as much as possible. The existing methods of ship drift path prediction only solve the problem of complex calculation, and it is better to predict the drift path of short distance. But in a long distance, the performance is not satisfactory. At the same time, the fitting degree and accuracy are not high. Precise prediction of paths is very important in helping to rescue and reduce casualties and there is a pressing need for improved methods to help solve challenging the prediction of the drift path problems.

For the study of ship drift paths on the sea, we need to consider influencing factors of ship drift. There are some existing methods for the prediction of the drift paths and the analysis of drift on the sea. Reference[1] used a probability model to predict the location and velocity of the drifting objects at each moment in the study of the drift trajectories of nonpowered ships, it obtained result that is the closest real speed of drift at a time. Reference[2] considered the influence of the natural environment factors such as the wind field and the flow field on the drift, and types of drifting objects were classified. If final location of the drifting object and the type are known, finally they could obtain the drift trajectory. Reference [3] proposed a random particle simulation method, which describes the influence of the uncertain and volatile marine environment and the wind pressure coefficient on the ship drifting. Reference [4] proposed Monte Carlo prediction method, it based on classical probability theory and mathematical statistics theory and stochastic process. Reference [5] based on the Leeway offshore drift theory and the Monte Carlo method. The experimental result was basically consisted with the actual results.

In this paper, we shall study the Monte Carlo method and then propose the particle reloading simulation method to predict the drift path of the ship. The rest of this paper is organized as follows. In section 2, we give the basic principles of Monte Carlo method. In section 3, we analyze the ship drift state and put forward a novel method that we call particle reloading simulation.In section4, the paper provides experimental results to validate the novel method. Finally, what is presented is the conclusion.

\section{MONTE CARLO METHOD}

Monte Carlo prediction is one of the most widely used methods in the drift path prediction. In the using of ship drift model, Monte Carlo method [6] simulates the ship drift process at first, and then calculates the initial probability distribution during the ship drift. Finally it solves the problem of ship drift path. Monte Carlo method bases on probability statistics, it regards the problem as a random event, and uses the random test to simulate the random feature of the problem of digital features, and the numerical features that obtained from the simulation of random events are considered as approximate solutions to the problem.

The drift model of the ship on the sea can be expressed by the following simplified formula (only considering wind drift and flow drift)

$$
x(t)-x_{0}=\int_{0}^{t} V(t) d t=\int_{0}^{t}\left[L(t)+u_{w}(t)\right] d t
$$

Where $L(t)$ is the wind drift, $u_{w}(t)$ is the flow drift, $x_{0}$ is initial drift distance.

The wind drift of the ship drift model is the drift that the ship is exposed to air and dragged or pushed by wind. Since the shape of the drift ship is not necessarily symmetrical, a wind 
pressure angle between the direction of the ship drift and the downward wind direction is caused by a lateral wind force, and the wind pressure angle is likely to occur on either side of the wind direction. According to the empirical formula, wind drift $L(t)$ is obtained as follows:

$$
L(t)=L_{d}=a_{d} W_{10}+b_{d}
$$

Where $a_{d}$ and $b_{d}$ are a linear parameter that obtained by a large number of fitting experiments. They are related to the type of floating ships. $W_{10}$ is the wind speed at $10 \mathrm{~m}$ of the sea surface.

In this paper, the numerical prediction of surface flow is realized by using the FVCOM model [7], it can be better to fit the complex curvature of the coastline by using the triangular unstructured meshes. In order to protect the energy conservation of the influencing factor, the finite volume method is adopted to process the physical quantities. The FVCOM model is not adequately considered on the temperature, salinity, density and so on. The equation of the FVCOM model is given:

$$
\begin{aligned}
& \frac{\partial u D}{\partial t}+\frac{\partial u^{2} D}{\partial x}+\frac{\partial u v D}{\partial y}+\frac{\partial u \omega}{\partial \sigma}-f v D= \\
& -g D \frac{\partial \gamma}{\partial x}-\frac{g D}{\rho_{\varepsilon}}\left[\frac{\partial}{\partial \mathrm{x}}\left(D \int_{\sigma}^{0} \rho d \sigma\right)+\sigma \rho \frac{\partial D}{\partial x}\right] \\
& +\frac{1}{D} \frac{\partial}{\partial \sigma}\left(K_{m} \frac{\partial u}{\partial \sigma}\right)+D F_{x}=u_{\omega}(t)
\end{aligned}
$$

The equation (1) is calculated by the second-order Longge Kutta method, and the equation (2) and the equation (3) can be taken to obtain the drift distance.

In order to solve the problem that the drift trajectory and the real trajectory drift with time, the Monte Carlo method is introduced to solve the uncertain factors and errors in the calculation process. The floating ship is defined as a single particle, and the drift trajectory of the particle meets the random process of probability distribution. Under the premise of no changing rules, it is assumed that motions of these particles do not affect each other, and the appropriate method is used to copy particles simultaneously. This is the idea of the drift model, which can obtain the drift regular distribution area of the particle over a period of time. This paper assumes that the process of drift ship is in accordance with the Markov process [8], the probability density distribution function of the next state of the floating ship is related only to the current state of the floating ship and not related to other factors. The random drift process is expressed by as follows:

$$
d x=V(x, t) d t+d \varepsilon
$$

Through the studying of wind drift and wind speed data can be obtained between about wind induced drift and wind speed regulation, but there are errors and accidental factors in obtaining wind speed and linear relationship, Therefore, the slope and intercept of the linear relationship need to add a random perturbation function[9], which satisfies the distribution $\mathrm{N}(0, \sigma)$. Wind drift coefficient is:

$$
\left\{\begin{array}{l}
a_{n}=a+\frac{\varepsilon n}{20} \\
b_{n}=b+\frac{\varepsilon n}{2}
\end{array}\right.
$$

When $W_{10}$ is $10 \mathrm{~m} / \mathrm{s}$, the slope and the intercept have the same perturbation,

$$
L=\left(\mathrm{a}+\frac{\varepsilon n}{20}\right) W_{10}+\left(b+\frac{\varepsilon n}{2}\right)
$$

Equation 6 is taken into Equation 4 to obtain the ship drift of the Monte Carlo prediction method.

Monte Carlo prediction method is to solve the low fitting degree of between the predicted drift path and the real drift path. It's solution is to exclude the effects of uncertain factors in the drift process. Firstly, it chooses two main factors (wind and flow fields), while the drift ship is treated as the particle, and the disturbance analysis is performed when the method calculates the wind field, flow field data and some uncertain factors are excluded as much as possible.

Although the Monte Carlo prediction method achieved good results, Monte Carlo prediction method does not solve to how to obtain real-time drift velocity in the drift process. While the method improves the accuracy of the drift path, but with the increase of the drift time, the error is still increasing. So we proposed a particle reloading simulation method based on the Monte Carlo method to solve the problem.

\section{Particle Reloading Simulation Method}

The first step of particle reloading simulation method is to determine the initial probability distribution of the drift ship in time and space, and then deduce the drift path. Different initial probability distributions come from different influencing factors on surface of ocean. It leads to a different drift path that affects the judgment of the final drift area of the ship drift. The particle reloading simulation prediction method can well describe the initial probability distribution of ship drift in complex environment. The path prediction of drift ship may appear multiple results. We assign them to different scenes according to certain rules, and obtain different initial probability distributions according to the different scenes. Then, we analyze the force state of particles and get the velocity of particle drift. At last, we can acquire the drift path. 


\section{A. Simulation Particle Drift State Analysis}

In this paper, drifting ship is transformed into a particle that has no influence each other, and they have the properties of floating ship and environmental forces. We need to analyze properties and force situation of ships, and then get the ship's force situation:

$$
\frac{(m+\dot{m}) d V}{d t}=\sum_{i=1}^{n} F_{i}
$$

By studying the drift ship, there are four main forces that affect the drift of the ship. They are the drag force of the wind, the radiation force of the waves, the drag force of the flow and the thrust of the turbulence. In this paper, particles are only affected by the above four forces. As for the intrusion ratio and the material of the ship, particles are automatically acquired, because they included above forces. According to the drift of the assumed condition, the acceleration of the ship drift model can be expressed as:

$$
\left\{\begin{array}{l}
\frac{(m+\dot{m}) d V}{d t}=\sum_{i=1}^{n} F_{i}=F_{a}+F_{w}+F_{c}+F_{t} \\
F_{a}=\frac{1}{2 C_{d \rho a} A_{a} W_{a}^{2}} \\
F_{w}=\frac{1}{2 C_{i w g} \rho_{w} L_{t} A^{2}} \\
F_{C}=\frac{1}{2 C_{c d} \rho_{w} A_{w} L^{2}} \\
F_{t}=\sqrt[2 R]{6 K_{a} \Delta t} / \Delta t^{2}
\end{array}\right.
$$

Where, $F_{a}$ indicates the drag force of the wind, $F_{w}$ Represents the radiant force of the waves, $F_{c}$ is the drag force of the flow, $F_{t}$ means the thrust of the turbulence, $\dot{m}$ originates from the acceleration of water particles on the surface of the drift ship. $C_{d}$ and $C_{c d}$ denote the drag force of wind and flow, $C_{i w}$ represents the coefficient of thrust of a wave, $\rho_{w}$ and $\rho_{a}$ respectively indicate the actual density of seawater and air.

Under normal conditions, drift velocity of particles can be obtained according to the following formula:

$$
v=v_{0}+\int_{0}^{\Delta t} a d t
$$

Where, $v_{0}$ is the initial drift velocity of the particle, $\Delta t$ is the time of the drift,a is drift acceleration of the particle.

\section{B. A New Path Generation Method Based on Particle Reloading Simulation}

We know the speed $\mathrm{v}$. Where $\mathrm{t}=\Delta t$, it is small enough. The method can form the integral curve, this curve is the drift path.

$$
y=x=x_{0}+\int_{0}^{t} v d t
$$

This method is predictive for short distance drift paths, but for drift predictions can produce errors on long distance. In this case, total time of the drift is divided into segments, and then particles are reloaded for each period of time. Each period will update initial probability distribution of the particle and force state of the particle at this time, which will make the drift path closer to the real state:

$$
y=x=x_{0}+\int_{0}^{t_{1}} v^{\prime} d t \int_{t_{1}}^{t_{2}} v^{\prime \prime} d t+\cdots+\int_{t_{n-1}}^{t_{n}} v^{n}
$$

After particle updates the information in N-1th time, $\int_{t_{n-1}}^{t_{n}} v^{n}$ is based on the force condition and the initial probability distribution to obtain the drift path in Nth time.

The particle reloading simulation prediction method is advanced for Monte Carlo prediction method. Firstly, according to the initial information of drift particles (mainly wind field, flow field, stress situation and floating material), the Monte Carlo method is used to obtain the initial probability distribution of particles. We select the drift time $\Delta t$ the particle, analyze the force and the state of the particle in time $\Delta t$, and obtain the drift velocity and direction of the particle according to above information. If the time of the particle drift is short enough, the method can obtain drift path in time $\Delta t$. If the time $\Delta t$ is relatively long, it will be divided into $\mathrm{N}$ parts. We repeat the process. Finally, at the end of each $\Delta t$, it is determined whether the particle initial information needs to be updated, then a new particle probability distribution is obtained. The particle drift analysis is performed again.

The algorithm of particle reloading simulation is as follows:

Input: initial information of the drift particle(including the wind field, flow field and its own attributes and other information); drift time;

Output: Drift path.

Step1: Enter or read the initial information of the drift particle to obtain the initial probability distribution;

Step2: Enter the particle drift time and analyze the state of particle drift;

Step3: determine whether the need to split the particle run time, if it is, it executes Step4, otherwise executes Step7;

Step4: the drift time is divided into $\mathrm{N}$ parts, sequentially update the particles in these time periods 
Step5: Output sequentially the drift paths in each time period;

Step6: determine whether the combination of output time is complete, if it is, it executes Step7, otherwise executes Step8.

Step7: Output the complete drift path;

Step8: exit algorithm.

The particle reloading simulation method divides the total drift time into multiple parts, and immediately updates the new drift state of the particle after generating a small drift path. According to the new state of the particle information, it generates a new drift path, which it will continue the entire drift process. The information constantly updated, the error continues to decrease. This approach makes the prediction of the entire drift path more and more close to the real drift path and achieves good results.

\section{RESUlTS AND Discussion}

In the experiment, the most typical inshore is chosen as a drift location. The inshore drift is the most frequent occurrence of accidents in recent years and it is representative.For influencing factors of ship drift,we choose the vector decomposition method.This section uses the Monte Carlo prediction method and the particle reloading simulation method for comparative experiments in this paper.

The starting point for this experiment is $\mathrm{S}(36.32 \mathrm{~N}, 120.73 \mathrm{E})$, it is the waters of the grass island. The end point is $\mathrm{E}(30.27 \mathrm{~N}, 117.35 \mathrm{E})$, It is the open sea of Mount Feng. In the experiment, the original drift trajectory is obtained.In fig1, the path is wavy because of the influence of periodic tides and ocean current.

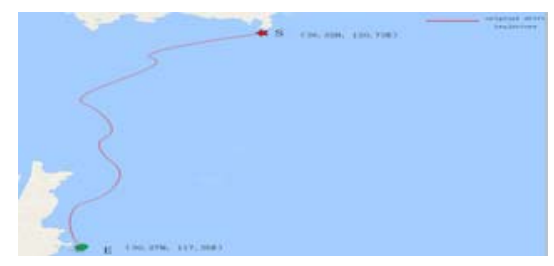

FIGURE I. ORIGINAL DRIFT TRAJECTORY

\section{Experiment:}

The vector decomposition method is the Monte Carlo prediction method and the particle reloading simulation method combined respectively. The influence factors of ship drift are dealt with by vector decomposition method.Two methods obtain the drift trajectory of the ship.We compare their fitting degree of drift trajectory with the original drift path fitting.

The red path is the original drift trajectory. The method 1 is the combination of vector decomposition and particle reloading simulation method, its drift path is black.The method2 is the combination of vector decomposition and Monte Carlo prediction method, its drift path is blue.

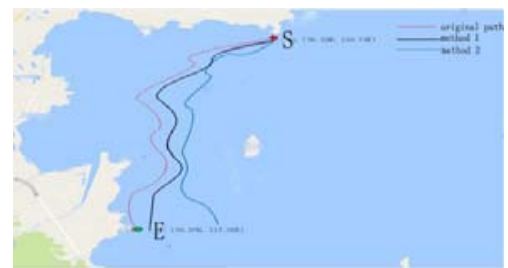

FIGURE II. THE PATHS OF THE TWO METHODS AND ORIGINAL DRIFT

In fig3, at the top the red frame is the initial stage of the drift; the black solid line is the middle stage of the drift; The dashed frame is the last stage of the drift.

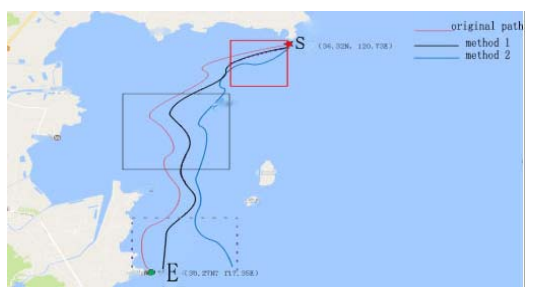

FIGURE III. THE ANALYSIS CHART OF THE DRIFT TRAJECTORY

In summary, it can be found that the drift path of method 1 and method 2 is basically consistent with the original drift path at the initial stage of drift. This is because of both the method 1 and the method 2 obtain the initial probability distribution of the ship drift model according to the Monte Carlo method, which has a great influence on the ship drift. In the middle and late stages of the drift, the fitting degree of the method 1 higher than method 2. Therefore, the particle reloading simulation method is more suitable for obtaining the ship drift path.

\section{CONCLUSION}

In this paper, the technique of obtaining the drift path of the ship is analyzed. Based on the analysis of the traditional method, this paper puts forward the method of particle reloading simulation. By decomposing the time in the drift process, the information is updated at the beginning of each time period to obtain the initial probability distribution of the ship drift. The drift path and the original drift path have a good fit degree. Two different methods of obtaining the drift trajectory of the ship are compared by experiment. The experimental results show that the proposed method has a higher fit degree with the original drift path.

\section{ACKNOWLEDGEMENTS}

This research is supported by

(1) 2017-2020 National Natural Science Foundation of China under Grand No.51679058

(2) 2013-2016 Higher Specialized Research Foundation (PhD supervisor category) of China under Grand No. 20132304110018

\section{REFERENCES}

[1] Allen A A, Plourde J V. Review of Leeway: Field Experiments and Implementation[J]. Review of Leeway Field Experiments \& Implementation, 1999. 
[2] Breivik.Fast ray sorting and breadth-first packet traversal for gpu ray tracing. Computer Graphics Forum, 2013,21(3):pp.132-137.

[3] Xiao F, Yin Y, Jin Y, Zhang X,. Determination of Maritime Search Area Based on Stochastic Particle Simulation[J]. Navigation of China, 2011, 34(3):pp.34-39.

[4] Zhang, Jinfen.Probabilistic modelling of the drifting trajectory of an object under the effect of wind and current for maritime search and rescue.Centre for Marine Technology and Ocean Engineering.2017,129:pp.253-264.

[5] Gao J, Lin M U, Wang G S, Cheng L I,Dong J X,Bao X W,et al. Drift analysis and prediction of debris from Malaysia Airlines flight MH370[J]. Chinese Science Bulletin, 2016.

[6] Zhong L, Sun Youchao. Research on System Maintenance Time Simulation Model Based on Monte Carlo Method and Roulette Rule[J]. China Mechanical Engineering, 2006, 17(10):pp.999-1002.

[7] Changsheng C, Beardsley R C, Cowles G. An Unstructured-Grid FiniteVolume Coastal Ocean Model (FVCOM) System[J]. Oceanography, 2006, 19(1):pp.78-89.

[8] Breivik ,Allen A.An operational search and model for Norcve ian Sea and the Nonh Sea.Journal of Marine Systems.2008.69:pp.99-113.

[9] Hu, Jian .Experimental and numerical study on wave drift forces on a semi-submersible platform in waves.Ships and Offshore Structures.2017.12(1):pp.56-65 